\title{
Synthetic Biology at the Limits of Science
}

\author{
Alfred Nordmann \\ In Bernd Giese, Arnim von Gleich, Christian Pade, and Henning Wigger (eds.) \\ Synthetic Biology. Character and Impact, Berlin: Springer, pp. 31-58
}

\begin{abstract}
What happens when some of the traditional questions and concerns of the philosophy of science are brought to the non-traditional field of synthetic biology? Given that synthetic biology is a very diverse field, this might serve to highlight the many ways in which it is business as usual. However, prominent concepts and research practices of synthetic biology can be seen to confound established ideas of how knowledge is produced and validated in the sciences. By highlighting and readying for discussion the tension between alternative images of knowledge production in synthetic biology, this paper seeks to open up debate among philosophers of science, and within the diverse community of synthetic biologists. With the advance of emerging technosciences like synthetic biology what is at stake is not primarily how they might or might not change the world. At stake, first of all, are epistemic values, the ethos and authority of science, and the relation of knowledge and power. Building on ongoing discussions, the paper begins by exhibiting contested notions of understanding, rational engineering, and design. In a second step, it turns to different conceptions of biological "systems" by presenting divergent accounts of the origin of synthetic biology and of how systems biology gave rise to synthetic biology. Finally, it seeks to focus the debate on a definition of synthetic biology, according to which it builds, for constructive purposes, on achievements of technical control of biological complexity, that is, that it uses these achievements to generate, rather than reduce, complexity.
\end{abstract}




\section{INTRODUCTION}

As in many so-called technosciences, some of the research practices and epistemic ideals of synthetic biology confound traditional conceptions of scientific method, regarding questions such as "how is knowledge generated and validated," "what does it mean to understand or explain something," "how important is the development of new theories," or "what is the difference between explorative experimentation and experimental hypothesis testing?" Indeed, on some accounts of what synthetic biology is and how it works, it does not even appear to be interested in traditional scientific methods of reducing complexity by intellectual means. Instead, it promotes the controlled generation of complexity by technical means, that is, by drawing available theories and tools into a technoscientific design process. 1

By inquiring how synthetic biology agrees with or confounds established notions of science, philosophers of science and of technoscience 2 contribute to a much larger process of delineating what synthetic biology is in comparison to other fields of biological research such as molecular biology, bioinformatics, systems biology, or genetic engineering. This is not a matter of classification or definition but of characterization: what are the basic assumptions, what are the routines and laboratory practices, what are the promises and ambitions, what is the special mind-set of synthetic biology? To the extent that it speaks with a distinctive voice at all, how does synthetic biology set itself apart from other endeavors? Given the fairly recent emergence of synthetic biology, there is already an impressive body of philosophical literature that adresses these questions (e.g., Bensaude Vincent 2013a and 2013b, Delgado/Porcar 2013, Gelfert 2013, Gramelsberger 2013, Kastenhofer 2013a and 2013b, Knuutila/Loettgers 2013, O’Malley 2009 and 2011, Schmidt this volume, Schyfter 2013).

\footnotetext{
${ }^{1}$ Here and throughout, the default meaning of "complexity" is simply that a structure or system is "not simple" or "difficult to conceive as a sum of simple processes" or "complicated." Science and classical theories of knowledge conceive the task of the human intellect as making sense of a bewildering multitude of sensory impressions by isolating from them simple patterns or lawful causal relations. This "reduction of complexity" is considered a major achievement of the mind. Accordingly, the first "limit of complexity" arises when things get to be too complicated to be tractable by the human mind (though a computer might still be able to achieve predictive control or to isolate strict causal dependencies). In contrast, the challenge of synthetic biology is seen as building up or generating complexity (the first sessions at the 2013 SynBio 6.0 conference were dedicated to the question of "realizing biological complexity," see the program under http://sb6.biobricks.org/, accessed January 5, 2014; also Mast/Möller/Braun 2013). Only at two points in the following discussion (see notes 10 and 16 below), does "complexity" assume a more exalted systems-theoretic status, thereby explicitly becoming a theoretical term. In the theoretical context of systems thinking, there is as second limit of complexity, namely irreducibility in principle. And only systems thinking thus conceived calls for an alternative, non-reductionist approach and thus a different kind of "reduction of complexity" - reduction not to aggregates of simple processes but to dynamic systems as integrated wholes.

${ }^{2}$ Many readers will not be familiar with the juxtaposition of science and technoscience as distinct modes of knowledge production. This is not necessary. The distinction will take shape over the course of this discussion as different ways of conceiving synthetic biology become aligned with the different epistemic values and ideals of science and of technoscience (Bensaude-Vincent 2009, Forman 2007, Nordmann 2010b).
} 


\section{FAMILIAR CONCEPTS, DIVERGENT MEANINGS}

By engaging claims of what synthetic biology can be and what it should be, philosophical scrutiny sharpens awareness, exhibits what is at stake, and thereby facilitates scientific controversy as well as public debate. Since synthetic biology is said to bring an engineering approach to biology, these debates concern how one needs to understand biological systems for the purpose of achieving technical control.

Three issues, in particular, stand out. They involve conflicting notions of understanding, rationality, and design. Though each of them deserves separate treatment, we will see that they are framed by different images of knowledge production, images that we will encounter also in the stories one tells about biological systems and the relation of systems biology and synthetic biology.

\section{(1) Creating Understanding}

The first of these issues is contained in Richard Feynman's oft-quoted statement "What I cannot create, I do not understand." ${ }^{3}$ This is a familiar issue in that it evokes philosophical positions that go back to philosophers as diverse as Thomas of Aquinas, Francis Bacon, or Giambattista Vico. ${ }^{4}$ Strictly speaking, it articulates a necessary, but not sufficient condition for what it means to "understand" something. It can be paraphrased as follows:

No matter how good our scientific models or our explanatory and predictive theories are, these are not sufficient for "understanding" as long as another condition has not been fulfilled. This necessary condition is the requirement that with the help of these models or theories, one can create in one's mind or in the laboratory the process or phenomenon in question. ${ }^{5}$

For philosophers of science, this formulation raises many problems ${ }^{6}$, but one thing is clear: According to this paraphrase, the ability to create appears as a crowning achievement, the final bit

\footnotetext{
${ }^{3}$ A review article speaks of the "repetitively, almost dogmatically, cited Feynman quote" (Rollié/Mangold/Sundmacher 2012). The source of the repetitively cited maxim is a photograph of "Feynman's last blackboard" which can be found, for example, at http://archives.caltech.edu/pictures/1.10-29.jpg (accessed January 3, 2014), compare e.g. Schmidt 2009.

${ }^{4}$ Aquinas argues that only God truly knows the world because he created it and one can only know what one creates (Aquinas 1986); Bacon declared that the power to control or to make things is a criterion of knowledge (which is why the statement "knowledge is power" is often attributed to him, see e.g. Pamela Smith 2004, 238-241); Giambattista Vico distinguished mathematics and the sciences of human culture from the natural sciences on the grounds that only the former truly know their humanly produced subject (Vico 1979). The notion that making implies knowing has been expressed also by scientists and engineers. However, it has not been uncovered as a proper undercurrent of methodological thought. For the pre-history of synthetic biology, Jacques Loeb's approach may have been particularly significant (Pauly 1987).

5 It is not clear why Feynman formulated this strong requirement on his "last blackboard." This may have been his objection to string theory in physics, or informed perhaps by his recent experience of discovering and demonstrating the cause of the Challenger space shuttle accident, see O'Malley 2009, 385-386.

6 The paraphrase suggests, for example, that understanding is more than the ability to explain and predict something. This prompts the empiricist suspicion that it is in this case too much to ask for scientific understanding. Also, if the material (re)production of a process or phenomenon is not the only way of creating what one seeks to understand, does the creation in one's mind require intellectual tractability as in a thought-experiment, or would a highly complex computer simulation also fit the bill?
} 
of evidence that proves all our previous thinking, our models and theories right. In the statement "what I cannot create, I do not understand", the notion of "understanding" remains first and foremost an intellectual notion that refers to science as an effort of gaining understanding through theories and models.

Now, when it comes to synthetic biologists, some adopt this strict and narrow interpretation of Feynman's statement. However, by embracing Feynman's statement to the point of treating it as a fundamental credo, ${ }^{7}$ synthetic biologists are expressing first and foremost that the seemingly opposing goals of human understanding and material construction can be jointly satisfied even as one brings an engineering approach to biology. If one wants to engineer a biological structure or process, one "cannot help" but gain understanding, also (Benner/Sismour 2005, 538 and 542). If this is the fundamental message, it is still an open question. However, what kind of understanding this is, and whether it serves as the capstone to theoretical knowledge production. Are synthetic biologists typically referring to the intellectually tractable, theoretical understanding that has been achieved, e.g. by systems biologists, and that is now ennobled and completed by efforts to actually create organismic structures? If only for the generally acknowledged large gaps in the explanation and prediction of biological phenomena, at least some synthetic biologists advance another way of paraphrasing Feynman's credo:

The ability to create or recreate biological entities or structures proves that we know enough to do just that, and the more dexterity we acquire the better we understand what makes these entities or structures work - even if what is known explicitly is only fragmentary and if it is complemented by much tacit and procedural knowledge, including technical know-how.

Unconcerned with Feynman's intention and unconcerned with the grammatical construction of "What I cannot create, I do not understand," this paraphrase inverts the formula to read "What I can create, I do understand." 8 The ability to create now appears as a sufficient condition for understanding, suggesting that "understanding" can leap ahead of explanation and prediction, and that it derives from a more immediate relation of knowing and making. To be sure, it is easy to

\footnotetext{
${ }^{7}$ Famously, Craig Venter and his collaborators encoded in 2010 the Feynman quote as an identifying watermark in the genetic code of the first chemically synthetized genome of a working bacterial cell. Notoriously, in so doing they misquoted Feynman ever so slightly.

${ }^{8}$ Schmidt 2009 warns that the inversion of Feynman's dictum does not follow logically from the original formulation. For examples of authors who adopt the second reading, see section 3 below. But see also the example of Alfonso Jaramillo who proved quite committed to the second reading during his oral presentation at the CAS Conference Synthetic Biology (July 23 to 25, 2012, at the Biocenter of the LMU, Munich). Arguing for automatic design, computational evolution, high throughput characterization he claimed for these methods that one does not need that much (theoretical) knowledge about structure to succeed, and that they allow quantitative testing with and in spite of limited knowledge. In published work this is expressed in a more muted fashion, more careful, in particular, to advertise this as a virtue of his approach: "As our automated methodology uses few specifications as inputs, it could also be used to test new mechanisms and hypotheses despite the lack of a complete molecular understanding of the living cell” (Rodrigo/Landrain/Jaramillo 2012, 15274).
} 
dismiss the second paraphrase as not being in line with Feynman's thinking, as being implausible from the start, or as being incompatible with the traditions and epistemic values of science (O’Malley 2009, 385-386). However, the philosophical analysis of synthetic biology cannot simply dismiss the second paraphrase but needs to reconstruct why it appears credible to those who maintain it. What is the "image of technoscience" that underwrites such an apparently "unscientific" conception of achieving understanding through making? What kind of learning takes place and what kind of knowledge is achieved if one submits to a program where one "cannot help but gain understanding" as one pursues a technical goal (Benner/Sismour 2005, 538 and 542)?

Here is one example, then, of questions for the philosophy of synthetic biology. After determining what is meant - in any given instance, but also in general terms - by appeals to Feynman's dictum, it will discover, undoubtedly, a diversity of interpretations and usages which pose the challenge of reconstructing their intelligibility. Here, it might discover that the more familiar and less problematic first reading of the dictum as offering a necessary "capstone"condition for understanding exaggerates and, idealizes the availability, scope, and power of explanatory theories in biology. At the same time, it may well discover successful design strategies that lend credibility to the seemingly more problematic notion of understanding a biological system while black-boxing mechanistic detail and without requiring intellectual tractability (MacLeod and Nersessian 2013).

\section{(2) Engineering Principles}

The second of the three issues concerns the familiar question of rationality, one that is typically understood by way of stark contrast. Either inquiry or engineering follow rational principles of construction and validation, or they are beholden to mere empiricism, haphazard tinkering, and exploratory experimentation. This either-or reflects a particular point of view, namely one that ranks rational engineering or rational design higher than strategies of trial and error. Accordingly, the distinction is often used to probe how far our knowledge of the world has advanced. Those who are still working in the mode of trial and error have not ascended as yet to a level of intellectual and technical control that would allow them to invent new processes or devices simply from considerations of theory and principle. Thus, the idea of "rational drug design" was advanced in the 1970 s by biomedical researchers who were scandalized by the notion that in this day and age drug discovery should proceed by way of randomized search procedures. And after trial and error - in the form of automated high-throughput methods - triumphed over rational drug design (Adam 2010), the scientific community came back with nanomedicine and visions of targeted drug-delivery ("this 
time we'll get it right"). Similarly, the aspirations of synthetic biology are often judged in these terms: Can the "synthesis" of biological structures or processes proceed in a planned, deliberate, theory- and evidence-based manner such that the intended outcome issues as if from a blueprint (Giese et al. 2013, O'Malley 2009, Gramelsberger 2013, Lewens 2013)? And, if this is not the case, is this only "not yet" the case, likely to become possible in just a few more years, or does it owe to a disciplinary style of doing things - with chemists seeking rational control while bioengineers are more comfortable with tinkering? Or does the failure of rational design owe to a limit of biological complexity that is irreducible and thus an insurmountable limit of control?

The hierarchical conception that places rational engineering above tinkering is blind to the possibility of rational tinkering. This is because scientific rationality is tied to calculability (Berechenbarkeit) and the ability to plan in advance, thereby tied also to the notion of natural law and the predictive abilities that flow from it. From an engineering point of view, however, rational engineering principles may well be opposed to blind groping but they are not necessarily opposed to search strategies that exploit random variations and thus trial and error. They are also not opposed to design strategies that involve iterative processes of adaptation and tuning. These, to be sure, are rational strategies by which to work around limits of knowledge, and to achieve technical solutions in the absence of information about mechanical detail. In other words, these are rational strategies to create robust black boxes ${ }^{9}$ or modules.

Quite in line with this engineering point of view, appeals to the "design cycle" are at least as frequent in synthetic biology as those to Feynman's dictum (e.g., Cheng/Lu 2012, Royal Academy of Engineering 2009, 18-23, Tabor 2012, UK Synthetic Biology Roadmap 2012, 13 ). Typically, the design cycle consists of three steps that are iterated until a desired technical performance is achieved. If the task is to create an informational technological expert system, or to create a climate model, the first step is to analyze the situation to be emulated, automated, or modeled. In a second step and on the basis of this analysis, a skeletal technical system is created. This first prototype is as far as the application of rational engineering principles will reach - it results from well-established procedures of mapping known features of the situation into a technical architecture. The third step consists in testing the prototype. Now, the performance of the prototype or model is observed and its ability assessed to emulate, automate, or model expert or climate behavior. At this point, any

\footnotetext{
9 The term "black box" refers to a technical unit of reliable functioning that is not and need not be scrutinized for the specific causal processes that would account for its functioning (Royal Academy of Engineering 2009, 19-20). Not all modules in a modularized architecture are black boxes, but black boxes can serve as modules (see, paradigmatically, Canton/Labno/Endy 2008). Black-boxing is the decision or strategy to create black boxes. Eran Tal (2013) offers a critique of the notion of black box and seeks to identify instead rational strategies that provide "ignorance affordances."
} 
discrepancy between the actual and the desired performance of the prototypical system induces a second, third, and further iteration. Each new iteration begins with another analysis, but this is no longer an analysis primarily of the original situation but now of the technical system, and why it does not yet perform as desired. On the basis of this analysis (step one again), modifications are introduced, and an improved prototype is created that may serve to better tune the expert system or climate model to the target system (step two). Some of these modifications draw on specific scientific knowledge of features that may have been neglected and are now added in. Other modifications draw on familiar engineering strategies, such as adding noise in order to dampen sensitivity, and yet others are simply tried out to see whether this or that may do the trick. All of these modifications of the behavior of the designed system are compared against the target system (step 3), and subsequently rejected or further modified. With each iteration new elements are introduced and the designed system as a whole gains complexity. In the limit, the designed system emulates the target system near-perfectly and does so because it is similarly complex. Thus one finds that a predictively successful simulation model can be nearly as complex and just as intractable as the "natural" system that is modeled by it (see, e.g., Lenhard/Winsberg 2010). This is a technical achievement by rational means. Though it does not consist in the application of "rational engineering principles," it is not "mere" tinkering either, but a strategy to systematically optimize the performance of a technical system.

For the philosophy of synthetic biology, the competing notions of rational engineering are of interest not only because they implicate the question of systemic limits of complexity: after all, ab initio rational engineering is possible only to the extent that calculability is even achievable. The different conceptions are of further interest because they speak to entirely different kinds of pursuit. On the one hand, there is science as an analytic enterprise which reduces complexity in order to arrive at principles which can be used to translate mechanistic accounts of biological processes into procedures of rational biological engineering. On the other hand, there is the technoscientific enterprise of synthetic biology which generates complexity by way of an iterative design process, that is, by way of a rational strategy to fine-tune engineered systems so that they can emulate biological systems. The philosophy of synthetic biology has thus to countenance at least the possibility of a research enterprise which seeks to exceed limits of intellectual tractability, of human understanding, or calculability; and does so in pursuit of technical robustness at ever higher levels of biological complexity. In this pursuit, the modules from which synthetic biology builds up 
greater complexity can be black boxes that work together in reliable ways. ${ }^{10}$ To be sure, when engineers stuff matters of detail and complexity into a black box and then compose larger technical systems out of input-output relations among these modular black boxes, they are not just building up but also managing or handling complexity - without claiming, however, that what formerly looked complex now appears to be merely an aggregate of so many simple relations. ${ }^{11}$

\section{(3) Intelligent Design}

The third issue for a philosophy of synthetic biology arises from the previous two, and is a classic question also for the philosophy of biology: At first, everyone believed that species were designed by their creator. Then Darwin contradicted this. So, how is it possible even to speak of creation and design in biology? While anti-Darwinian theories of intelligent design are in ill-repute, how can synthetic biology speak of design processes and simultaneously take the insights of evolutionary biology into account?

Darwin showed that biological entities and processes are products of natural history and not of design. When synthetic biologists now get into the business of producing them by design, they are not thereby denying the ubiquitous and powerful action of evolution by natural selection upon anything that is subject to variation or less-than-perfect replication. On the assumption that they are interested in maintaining the continuity between the scientific naturalism of Darwinian biology and their engineering-oriented enterprise, synthetic biologists are therefore engaged in a philosophically significant effort. Implicitly and explicitly, conceptually and practically, they establish the compatibility of evolutionary and synthetic biology. This effort consists firstly and primarily of isolating the design efforts from evolutionary processes - be it by studying all organisms as if they were humanly engineered ${ }^{12}$, be it by limiting the work of synthetic biology to closed industrial

\footnotetext{
${ }^{10}$ Note that for the analytic enterprise of science, the issue of calculability is central and, by the same token, the nature of complexity or of emergent properties. Science seeks to know whether biological structures and processes are irreducible in principle or subject, sooner or later, to an analytic reduction of complexity. In contrast, the technoscientific interest in generating complexity is quite indifferent to this question. Perhaps, new and irreducible systems qualities emerge over the course of iterating the design cycle, perhaps not. No matter how one conceives the "limits of complexity," the design process aims to overcome them (compare note 1).

${ }^{11}$ This important qualification owes to comments by Maureen O'Malley. "Reduction of complexity" usually and in this text refers to an intellectual achievement: Complex phenomena can be reduced to simple processes and their aggregate effects. But some speak of a different kind of reduction of complexity: "synthetic biologists simplify and build" (Ferber 2004, 158, also Calvert 2010). Whereas systems biology seeks total information and thus incorporates into its representational models all the findings of omics-research, synthetic biology wants to find out how far we can get with what little we know - it does not try to incorporate as much information as possible into the process of generating biological complexity. Synthetic biology attempts to find technical means which afford ignorance (Tal 2013), allowing it to succeed with less information rather than more. This might be considered synthetic biology's technical "reduction" of complexity.

${ }^{12}$ In the terms of Daniel Dennett, after rejecting that natural organisms are the product of design, one can adopt a design stance towards them and studying them as if they had been engineered.
} 
processes, be it by adopting design constraints that prevent replication, variation, or interaction with biological systems, or be it by downplaying the likelihood that synthetic biology might alter the course of evolution. Each of these approaches raises questions of its own. What holds true for all of them, is that by conceiving the same biological entity at one time as an object of design (as far as that will go), and at another time as an object of evolution (to the extent necessary), synthetic biologists are tending to the boundary between organism and artifact even as they appear to undermine or even reject it.

A second dimension of the relation between design and evolution comes in when the trial and error aspects of the design process are analogized to variation and selection. Variations are introduced more or less randomly into the design cycle and the resulting system behavior is then selected for, or selected against, in a process that adapts performance to expectations (e.g., Bujara and Panke 2010). Indeed, if the aim of synthetic biology is to generate complexity, it may well appear as if the goal was to reproduce the work of natural evolution, albeit in a more accelerated and more purposeful manner. At first sight, this would give license to saying that synthetic biology is biomimetic: that it merely seeks to emulate or imitate nature, and for that reason, that it is presumably more or less benign. This analogy is haunted, however, by the same problem that confronted Darwin's analogy between artificial and natural selection, namely that there is place for a benign purpose, for a breeder or creator only in artificial selection, and not in natural history. Accordingly, strategies of trial and error that select proposed variations by way of performance criteria should be likened to artificial selection and breeding, not to natural selection and evolutionary history.

With the ambition, however, to reproduce the work of natural evolution in a more purposeful manner, the problem of technological hubris begins to raise its head as the biological engineer is likened to the divine creator that was banished from the modern scientific worldview (cf. Schummer 2011, 190-210). This ambition owes to a popular notion that preceded and accompanied the appearance of synthetic biology. This is the notion that in the development of human culture we are ("finally") reaching the stage where we can take evolution into our hands. This notion implies not only that humanity is now fully in command of its own destiny, it implies also that we are no longer subject to the haphazard, cumbersome, and often inefficient ways of evolution (cf. Dyson 2007). This is different from worrying that synthetic biologists are "playing God," for, how could they, if there is no God in a scientific account of nature? It is different also from looking at nature mechanistically in order to discover principles for the construction of mechanisms. Instead, this is a view that considers "nature" an engineer of sorts, one of us and one like us, who is in the business 
of designing biological artifacts and whose creations are considered to be wonderfully subtle and intricate but also as a bit round-about, full of redundancy, and perhaps unnecessarily complicated. As an engineer, nature is constrained by evolutionary history, by the relative fixity of species, and a small range of variations. In contrast, synthetic biologists or genetic engineers are not limited by lineages and the restrictions they impose on the gene-pool. This gives them the significant advantage of not having to work as slowly and conservatively as evolution by natural selection.

Once one arrives at this image of technological hubris, a last and perhaps most remarkable fact about synthetic biology needs to be countenanced, namely the near-absence in the scientific literature and in review articles of explicit discussions of synthetic biology's possibly problematic relation to evolutionary biology. ${ }^{13}$ But surely, there must be an explanation for this and perhaps the fault lies with those who see the need for this discussion. On the whole, perhaps, synthetic biologists need not worry about the consistency of the scientific world-view of evolutionary biology and their own technoscientific mind-set. Molecular biology has shown that one can engage in structural investigations without immediate reference to theories of evolution. Not unlike engineers in other fields, it is proving quite sufficient for molecular and synthetic biologists to work along pragmatic lines: "If there are laws of nature, we can't violate them anyhow, and in the meantime, it is our job to push the limits of technical possibility." In other word, attempts to probe what can be done by way of creating biological entities and processes do not advance ideas that need to be fitted into a larger biological world-view; instead, they merely find themselves more or less constrained by some general facts of nature (Nordmann 2010a).

The very questions of how biological engineering should be related to natural history, or of how evolution by natural selection differs from the design of biological artifacts thus depend on our conception of synthetic biology - is it an intellectual enterprise with at least some theoretical ambitions or should one judge its attempt to advance understanding of biological systems only in engineering terms? Only in the former case does the problem arise of having to reconcile the competing ideas of evolution and design, while in the latter case it is merely a practical challenge to insulate as far as possible the construction of biological entities and processes from the vagaries of evolutionary influence. This fault line between synthetic biology as somewhat theory-oriented and

\footnotetext{
13 The place where evolutionary considerations are most likely to appear is in "what if" scenarios that begin by valorizing synthetic biology and portraying its success at creating artificial organisms. Only then the question is asked what will happen once these are subject to evolution - either by way of "mutating" from benign to dangerous organisms, or by way of their ability to outcompete natural organisms, changing the make-up of biological diversity, and the like. The engagement with evolutionary concepts thus tends to begin only when synthetic biologists look at the potential impact of their work through the perspective of technology assessment. Arguably, though, it should enter in right at the beginning of their work, in reflections on the rhyme and reason of naturally evolved biological complexity.
} 
as exclusively engineering-oriented also separates the two ways of paraphrasing Feynman's dictum as well as the two conceptions of rational engineering. It is the fault line that runs between science and technoscience.

\section{FROM SYSTEMS BIOLOGY TO SYNTHETIC BIOLOGY}

So far, we have been considering only conceptual issues that resonate with familiar discussions in the philosophy of science and that receive another turn of the screw through the contemplation of synthetic biology. These have drawn our attention to the general scientific or technoscientific character of synthetic biology which, in turn, refers us to its history. Promoters and observers of synthetic biology position it on the one hand in respect to the history of the biological sciences, and on the other hand to the prospects of biological engineering, and to biology as a technoscience. In particular, they position it in respect to systems biology that may have laid the groundwork for the appearance of synthetic biology. But as to how, and to what extent, there are different stories that can be told. Of these, only two will be juxtaposed here. ${ }^{14}$ The first treats the move from systems biology to synthetic as the consequence of a paradigm-shift or a whole new chapter in the history of the biological sciences, one that revolves around systems-thinking as the best way of coming to terms, intellectually, with complexity. The second treats synthetic biology as a technoscience that considers systems only as more or less efficient units of technical functioning, and that goes beyond our simple intellectual ways by seeking the means for generating or increasing complexity. ${ }^{15}$

\section{(1) Sublime Thinking}

The first of these stories underwrites a comprehensive report that was commissioned by the German Ministry of Research BMBF and that gave rise to the present volume. It goes as follows. Biology has run up against the limits of complexity as it has tried to become a lawful and predictive natural science in the guise of molecular biology. Under the heading of "evo-devo" this was pointed out by a coalition of biological researchers and philosophers of biology. Processes of self-organization, laws of form, the reciprocal relations between a biological entity and its environment - all these were thought to elude the grasp of a physico-chemical methodology that needs to isolate and control

\footnotetext{
${ }^{14}$ For a sketch of a third story, see note 20 below. - Like all myths of origin, these three are idealized to the point of caricature, and they are told for reasons not of descriptive accuracy but of the moral they contain. Each in its own way has normative implications, suggesting what synthetic biology ought to be and what opportunities and risks it poses, what obligations and expectations come with it.

${ }^{15}$ Following upon and adding to the section on "familiar concepts, divergent meanings" the two stories might be said to expose the divergent meanings within synthetic biology of the notion of "system."
} 
specific causal processes as much as possible. There appeared to be only one way for biology to move forward and to become predictive or even constructive. It had to take biological complexity seriously, that is, to understand biological structures at least from the cellular level upwards as systems that exhibit the dynamics which are the subject of a general system science or a theory of non-linear complexity. ${ }^{16}$ This way of "learning from nature" led to systems biology which, in turn, prepared the ground for synthetic biology which, on this account, can be understood as applied systems biology: The processes of self-organization that are the subject of systems biology are applied in synthetic biology to the task of engineering biological structures. Inversely, synthetic biology can be said to contribute to basic biological science in that it constructs and exhibits structures and systems for study.

A kind of paradigm-shift within science thus becomes a paradigm-shift for engineering, too (Schmidt, this volume). Just as the science of biology has moved from causal analysis by physicochemical means to the identification of dynamic patterns through systems thinking, so bioengineering is moving from the science-based construction of genetic blueprints to synthetic biology as a form of engineering that harnesses self-organized growth for the creation of novel artifacts. Accordingly, the most prominent risk of synthetic biology is the release of synthetic organisms, and the disruptions these technologically evolved structures might cause in naturally evolved systems. By the same token, our best protection is awareness of the sensitive dependencies of complex systems - if synthetic biologists avoid making their constructs too robust, or avoid making them independent of very specific environmental conditions, all might be well (e.g. Schmidt 2009, 96-97). Complexity demands respect and this respect, in turn, might assure the proper fragility of artificial organisms that will not be able to survive outside the very special contexts for which they were synthesized or grown.

This first story of the rationale for systems biology and its application in synthetic biology is normative in that it demands that the research by synthetic biologists properly applies systems biology. ${ }^{17}$ This takes the form, for example, of maintaining that synthetic biologists ought to incorporate noise into the design process - not as something that needs to be minimized, corrected for, or excluded; but as something that in a proper understanding of biological systems is an essential feature of any self-regulatory biological system, natural or engineered. How one deals with

\footnotetext{
16 Here, then, "complexity" becomes a theoretical term that differentiates complex systems from merely very complicated aggregates of simple processes (see note 1 above).

17 This normative insistence on proper systems thinking extends the debate within and about systems biology (Wolkenhauer/Mesarovic 2005, O’Malley and Dupre 2005).
} 
noise (as a disturbing factor or as essential element) is thus said to betray whether one is or isn't truly engaged in systems thinking (Schmidt 2014, Gleich et al. 2012).

\section{(2) Technical Opportunities}

The first story ended on a note of suspicion. Despite its being called "systems biology," it appears unclear what is meant by "system" here. Does one mean a dynamic structure that requires general systems theory or a theory of non-linear complex dynamics to describe it, or does one mean a technical construct that consists of at least several interacting parts?

If it turns out that many or most systems and synthetic biologists do not aspire to a holistic way of thinking, a second story can be told. It is a story of technical opportunism according to which the concepts, theories, and methods of biology, biochemistry, and genetic engineering become absorbed into an engineering idiom. This second story does not begin with philosophical insights about a non-mechanistic type of causality, about the profound difference between organism and mechanism, about biological complexity and systematic limits of molecular biology. Instead, it begins with the lessons learned from the Human Genome Project. On the one hand, the project represented a triumph of analytic methods, having been completed sooner and more efficiently than anticipated an achievement that continues as genomics produces cheaper and faster methods by the year, if not by the month or day. On the other hand, it delivered a blow to straightforward genetic determinism in the sense that only very few single genes can be correlated to single traits. This insight prompted neither retreat nor profound reorientation, however, but an attitude of "offense is the best defense." If the causal determinants of dispositions, traits and also of disease are far more complicated - and "complex" only in this sense - one needs to expand the tool-set developed for the Human Genome Project, and for that one requires the accumulation of yet more data, trusting that new insights and tools will be generated by the ever-improving technologies for the representation and processing of large data-sets. This data-fetishism and the many kinds of "omics" proved pervasive in the funding and organization of research, even without enjoying much intellectual prestige. The mere accumulation of data and the race to fully map genomes and proteomes appears rather pedestrian, and this is where systems biology comes in. It provides a kind of format and form, rationale and rationalization for the idea of "total information" and its accumulation. This rationale comes from the idea that one might model whole structures and organisms by integrating as many data as possible and by approximating a complete description of a biological "system" (which is nothing more on this account than an aggregate of very many components and causal pathways). 
Since the computer served not only as the tool but also as the site of much systems biology research, it became apparent that computers are far more than devices for the storage and organization, representation and modeling of data. Computers are physical systems in their own right that can instantiate dynamic processes such that the behaviors of data-systems can be created, modulated, and observed, such that input-output relations can be studied, such that control can be achieved and stabilized even as the particular causal pathways remain opaque. It is this fact that leads from systems biology to synthetic biology in the second story. ${ }^{18}$ Here, systems biology comes first and takes priority only because it integrates a multitude of data in order to represent biological systems such that they can be studied and understood. Synthetic biology comes second and shortcircuits the ambitions of systems biology: Where the latter produces representations, synthetic biology takes these as substitutions, that is, it regards model systems that are subject to modulation and control in silico as a prototype for the construction, modulation and control of biological systems. Thus, while systems biology begins by capturing complexity and rendering it for the purpose of reducing complexity through theoretical modeling, synthetic biology does not demand theoretical understanding and the reduction of complexity but has learned from systems biology that complexity can be generated in a controlled manner. ${ }^{19}$

Though synthetic biology shares many of its ambitions with genetic engineering and other fields of molecular and bioengineering, it is engineering not by way of creating a knowledge-based hypothesis-driven blueprint of how things should work, and then implementing it. As suggested above, it does not work in the mode of rational engineering. But it is also far more than mere tinkering, trial and error, and the development of automated high-throughput search strategies though these have a role to play. Synthetic biology is opportunistic by asking strategically how much technological knowledge and control one can achieve with what little we know scientifically,

\footnotetext{
${ }^{18}$ Computation for systems biology enabled better ways to "acquire, store, analyze, graphically display, model, and distribute" information. Without yet going there, the discussion of computer models in systems biology prepares the ground for the exploitation of what they afford in terms of performance, behavior, intervention and construction (Ideker/ Galitski/Hood 2001, 347-353). This holds also for that brand of systems biology that takes complexity seriously. Here the proposals by Kitano (2002 and 2004), for example, mark the point of transition. He advocates engineering concepts and computing tools for the purposes of modeling, representation, and theoretical understanding of biological complexity. He thereby paves the way for modes of constructing and handling such systems without reference to complexity theory: his concepts and tools afford their employment towards constructive ends by synthetic biology (O’Malley et al. 2008, 62). - This point of transition is also discussed by Schmidt (this volume). He sees bioengineers who adopt systems thinking. The story of technical opportunism sees systems thinking appropriated and vulgarized by the ordinary idiom of engineering (Nordmann 2010a).

19 Gabriele Gramelsberger identifies the simulation approach as a common denominator of systems and synthetic biology and suggests that it provides rational design methods that support tinkering in the lab (Gramelsberger 2013). She thereby downplays that modelling in systems biology is said to be "for basic research (i.e. generating knowledge) whereas synthetic biology's modelling is for the design of constructs" (O'Malley et al. 2008, 62): "Ultimately, mathematical models developed for research purposes (e.g. in systems biology) will be employed as design models in synthetic biology" (Heinemann/Panke 2006, 2796).
} 
finding that through an iterative design process one can achieve a great deal. The IGEM competition pursues this strategy most overtly as a proof of concept that US undergraduate students can "do" synthetic biology (Check 2005, see also Dyson 2007, Delgado 2013). This type of engineering is inspired by software engineering, for example, by the creation of expert systems which tune models to performance parameters, enriching the models until they achieve the desired functionality. According to this story, the impact of synthetic biology is first and foremost on the culture of research and the way of doing science itself. And the "risks" associated with synthetic biology concern societal expectations of the kind of knowledge and experience that is needed for the adoption, assessment and regulation of technologies; they thus concern our tolerance for blackboxed processes. This has been discussed, for example, in respect to the "kludge" as a module in a large software program or in an engineered assembly of biological pathways which plays an unknown, yet apparently necessary, role for the correct functioning of the system (O'Malley 2009, 2011, compare Lenhard/Winsberg 2010).

\section{(3) (Techno)Scientific Biology}

Having heard first the story of sublime ascendance to systems thinking in engineering and then the story of technical opportunism for building up un-theorized complexity, it is finally important to reflect on the juxtaposition of these stories or the starkness of their opposition. ${ }^{20}$

There is a strong temptation to believe that the two stories about the relation of systems biology and synthetic biology are easily reconciled, that they are but two sides of the same coin (Breithaupt 2006, compare Kastenhofer 2013a, 2013b): Synthetic biology advances profound theoretical understanding of biological systems even as it opportunistically pursues an engineering approach to the design and creation of biological entities and processes. This would amount to denying that there are profoundly different ways of conceiving synthetic biology. And to the extent that there is a philosophical difference to speak of, it would appear to be one that has been rather familiar since the times of Kant, namely the tension between a holistic understanding of organisms and the mechanistic materialism of modern science. It might be sufficient - and this would be an argument for reconciliation and business as usual - that synthetic biology is dedicated to theoretical

\footnotetext{
${ }^{20}$ There are other stories that could be told. One does not have to assume that synthetic biology is somehow derived from, or intimately related to, systems biology. Instead, one might foreground the relation between chemistry and biology as exemplified, for example, by the work of Steven Benner (Benner/Chen/Yang 2011). Just as physicists were told, many years ago, that there wasn't much work to be done in physics anymore but that they might find interesting problems in biology, so chemists have been told a similar story in recent years (I owe this suggestion to H. Ulrich Göringer). On this account, it is the chemical approach that distinguishes synthetic biology and genetic engineering. The possibility that the "synthetic" in synthetic biology derives from synthetic chemistry was discussed by Bernadette Bensaude-Vincent (2013b, compare 2009b and 2009c).
} 
understanding as well as the constructive project of building up ever more complex biological structures. It sometimes does so in the name of an ambitiously holistic notion of "system" and sometimes in reference to a rather more mechanistic conception of a system as a complicated unit of technical functioning.

Against this proposed reconciliation and the notion that synthetic biology can have it all, the present analysis suggests that there is no easy way out. If there is anything different and new about synthetic biology, it may well consist in the way it challenges the traditional orientation of the biological sciences and even of biotechnological research. Indeed, it would be a misunderstanding of the juxtaposition of the two stories about systems and synthetic biology if one took it simply to rehash the contrast of irreducible holism vs. reductionist mechanism. Instead, the debate of holism vs. mechanism belongs altogether to the first story which is driven by theoretical concerns and debates. According to the first story, systems and synthetic biology constitute a paradigm-shift of sorts, and it is entirely within that story that anti-reductionist "systems thinking" prevails over attempts to reduce biological phenomena to deterministic causal relations that can be isolated in the laboratory or in the mind.

If the debate between different intellectual conceptions belongs to the first story, it is characteristic for the second that the clash between competing research paradigms and all its attendant questions fade away and become irrelevant. Questions of reductionism, of natural philosophy, or the fundamental difference of organism and artifact are of no concern to the technical opportunism of synthetic biology. These questions are neither answered nor dismissed, but merely absorbed into an engineering idiom (Nordmann 2010a). The engineering approach of synthetic biology is not holistic or engaged in systems thinking as it builds up complexity in a controlled manner through iterations of the design cycle (pace Schmidt, this volume), but it is also not mechanistic. Likewise, it does not challenge in a profound or principled manner the difference between artefact and organisms as it constructs a robust black box which, in its opacity, is not at all unlike the biological organism as a black box with stable behavioral patterns.

To put it a bit metaphorically, then, in relation to systems biology, the two stories about synthetic biology do not attribute to the researchers different theories, opinions, or beliefs but an entirely different mind-set, a different way of living the laboratory life, of participating in history and relating to the tradition of science and the Enlightenment. On the one hand, there is the scientific mind-set of those who query the limits of reductionism and embrace systems thinking; on the other hand is the technoscientific mind-set of those who no longer seek the most appropriate way of 
reducing complexity and promoting intellectual understanding, but who proceed instead to generate biological complexity from available theories and techniques.

\section{SCENES OF CONFLICT}

We first saw the fault lines between scientific and technoscientific orientations of research that separate different notions of understanding, rationality, and design. We then saw how these fault lines provide contour and organize the stark juxtaposition of two powerfully coherent stories about the origin of synthetic biology and its relation to system biology. The easy way out would be to blur these boundaries and vaguely have it all. By blocking this easy way out, the terrain has been laid out. In a third step, we can now observe how researchers position themselves in this terrain. Though it is possible to wear different hats at different times-in one context advancing theory development and the reduction of complexity, in another context promoting the design of highly complex entities - this does not hold for publications that each belong to just one context. Any given publication expresses only one mind-set, exhibits one research agenda, establishes one kind of relation to its object. So, even if researchers might not position themselves unambiguously, every particular publication can be assigned a definite place on the map.

Different researchers engage in different research practices, and by looking at the published products of these research practices we can see how the same researchers can belong to very different epistemic communities in that their work is informed by different values, methods, standards of evidence and criteria of success. ${ }^{21}$ Therefore, questions, issues, and hypotheses can be sharpened by treating the publications of synthetic biology as scenes of conflict between the values of the different epistemic communities. In conclusion then, we might cast a brief glance at three such scenes of conflict.

\footnotetext{
${ }^{21}$ Arguably, the initial promise and attractiveness of synthetic biology is much like that of nanotechnology. However, the clash between epistemic communities is far less pronounced in nanotechnology than in the case of synthetic biology. Nanotechnological research is "pure technoscience" because it is geared to the development of basic capabilities of control that generally expand the toolset of technology - it isn't dedicated to any one engineering agenda but seeks to recruit scientific theories, scientific expertise, scientific labor for the purpose of putting technological change on a new footing. Nanotechnology is thus an effort to retool the scientific enterprise by dedicating the accumulated knowledge, methods, and personnel for knowledge production to a different, perhaps complementary end. Synthetic biology is "pure technoscience" in a different way. It does not seek to retool or rededicate laboratories and academically trained researchers. Instead it seeks to produce new kinds of researchers even before it produces new kinds of biological entities. The creation of epistemic communities with non-traditional values is part of what synthetic biology is and, for some of its protagonists, what it ought to be. The promise and attractiveness of synthetic biology thus lies also in its appeal to a new generation of researchers. This is somewhat problematic, however, since the staging of a generational conflict over epistemic ideals does not go so well with the idea of drawing together a diverse group of researchers.
} 


\section{(1) Accommodating Ignorance}

One does not have to cast far and wide to find scenes where different epistemic and, indeed, generational communities clash in the field of synthetic biology. This occurs at any conference where senior researchers confront so-called iGEM teams that impatiently seek to achieve on extremely short time-scales what others frame in terms of multidisciplinary, sometimes career-long research trajectories. This discrepancy of expectations cannot be ascribed simply to naïveté on one side and many years of experience on the other. The iGEM teams seek to find out through a strategic design process how much they can achieve with what little they know. They are not held back by seeking to learn all that would be needed for rationally engineering some biological structure or entity. Instead, they are invited and resolved to short-circuit the scruples of their teachers. If science is about the search for knowledge in order to reduce specific areas of ignorance, iGEM's technoscientific approach acquires a kind of working knowledge that can work around and accommodate ignorance.

Such scenes of conflict rarely take the form of overt disagreement, opposition, or antagonism. As with the encouragement of iGEM teams also within relatively conservative departments, they can involve something like the turning of a blind eye to the differences. An issue of Nature dated January 21, 2010 provides such a scene of conflict. Its featured article, Roberta Kwok's “Five Hard Truths for Synthetic Biology," offers a review of five major obstacles to the ambitions of synthetic biology and the prospects for overcoming them.

The text begins, predictably enough, by pointing to the "daunting knowledge gap when it comes to how life works" and, quoting Christina Agapakis, to the fact that "there's a lot of biology that gets in the way of the engineering." It goes on to show, however, that synthetic biologists are undaunted by the daunting knowledge gap and that they might have ways to meet the key challenges which they encounter all along the way. The first difficulty of defining standardized biological parts provokes efforts to substitute relative for absolute measures, thereby taking a first stab at evading Martin Fussenegger's verdict that " $[\mathrm{t}]$ his is the type of complexity that is very difficult to capture by standardized characterization." The second difficulty is the familiar predicament that predictable design procedures are not available, whereas trial and error is too arduous. Here, the synthetic biologist's answer is said to consist in a process of directed evolution, i.e., the design cycle and its iteration "until the system is optimized." The third difficulty arises as one moves to ever greater levels of system complexity which prompts the proposal to overcome the bottleneck by automating the process quasi-robotically, or by using bacteria as assemblers. In order to avoid unexpected interactions - the fourth difficulty - procedures need to be found to insulate the 
biological machinery to be designed as far away as possible from a cell's "natural machinery." And the final difficulty is to avoid variability and to increase stability, for example, by using noise to one's advantage rather than try to eliminate it. Accordingly, the review closes on a note of cautious optimism that synthetic biology can move forward without closing the daunting knowledge gap:

"As the cost of DNA synthesis continues to drop and more people begin to tinker with biological parts, the field could progress faster, says [Rob] Carlson. 'It's a question of whether the complexity of biology yields to that kind of an effort."' (Kwok 2010, 290)

In summary of Kwok's arguments, then, she argues on all five points of difficulty that the only chance for synthetic biology to succeed is by way of design processes that can accommodate or work around ignorance.

But how do the editors of Nature relate Kwok's assessment of the first decade of synthetic biology? On the tenth anniversary of the repressilator and a switchable regulatory network, they declare that "[c]ontributions to and from basic science are the part of synthetic biology that most deserves celebration":

"Both of those pioneering experiments transposed two great traditions of physics to biology: first, to understand something one must build it, and second, start from the simplest imaginable principles. These directives have set the basic-science agenda for synthetic biology: to design, and thus define, the minimal systems sufficient to produce a given function. [...] Bringing these applications to reality has proved much harder than was originally hoped (see [Roberta Kwok's analysis]). But the difficulties have proved instructive. Indeed, the decade-old papers raised several new and fundamental issues in biology, for example by pointing to the crucial role of noise in gene expression, both as a nuisance and as a great computational opportunity. It is now an active area of research. [...] It took endeavours in synthetic biology to illustrate what systems biology perhaps should mean: to enlist mathematical formalism in producing biological insights that are beyond the reach of mere intuition." (Nature editorial 2010)

The editors thus try to assimilate Kwok's analysis into the traditional idiom of basic vs. applied science. This is the vain attempt to accommodate the epistemic ideals of a technoscientific design community within that of traditional science. In which sense, for example, is the design of the repressilator an "experiment?" Is "to understand something one must build it" really a principle of physics as a basic science? How and when is "to design" the same thing as "to define" - even if one considers operational rules as definitions, what is defined by the complete "design" of a minimal system? What makes the synthetic bottom-up design of a minimal cell preferable to the classically analytic "knock-out" methodology if the aim is to discover the contribution of individual genes to the workings of a cell? And, finally, the editorial states that biology moves beyond the reach of 
mere intuition when aided by mathematical tools. Does this not imply that knowledge or understanding now reside in the ability to build a computer model, rather than in theories that are tractable by the human mind? ${ }^{22}$

Here, then, the scene of conflict appears as an unresolved tension within a pair of texts that does not wish to acknowledge, and turns a blind eye to the profound difference in the conception of a research field that contributes to basic biological science and one that pursues a knowing-bybuilding design agenda.

\section{(2) Discontinuous Continuities}

In their 2010 paper on "Engineering in Complex Systems" Matthias Bujara and Sven Panke also produce an argument that explores the tension between different epistemic communities, those of knowledge-based rational design and those of "evolutionary" design that feeds variation and selection into the iterations of a design cycle. ${ }^{23}$ They distinguish three types of optimization strategies, showing that designers can work more effectively if they know what they are looking for, that is, what to vary and what to select for:

Random evolution based on mutagenesis and brute force screening only requires limited knowledge on [sic] the system but the cause for the beneficial effect frequently remains unclear. Directed evolution needs at least knowledge of the element that should be modified (e.g. gene, promoter, or ribosome binding site), while a detailed optimization strategy is not needed. Combinatorial design follows a semi-rational strategy based on characterized parts and compensates for the current lack of detailed instructions for a comprehensive blueprint for optimization. $(2010,589)$

The progression from "random evolution" to "combinatorial design" thus involves an increase of knowledge about the elements to be modified and the functions to be achieved which is illustrated by a very telling graph that extrapolates from the three "evolutionary" strategies all the way to rational engineering.

\footnotetext{
22 The editors' text continues here in agreement with the story of technical opportunism which was told above and which contradicts the idea that synthetic biology advances basic science: "In that aspect [of using mathematical formalism to manage data beyond human intuition], synthetic and systems biology now seem indissociable."

${ }^{23}$ See section 1.3., above, as to why "evolutionary design" is an oxymoronic misnomer. Though they both work with variation and selection, Darwinian evolution by natural selection is different from breeding by artificial selection: what is selected for and against in natural selection does not depend on the specifications of a designer, but on adaptedness to the complex and changing conditions of life. What Bujara and Panke are referring to is more appropriately called "design by breeding."
} 


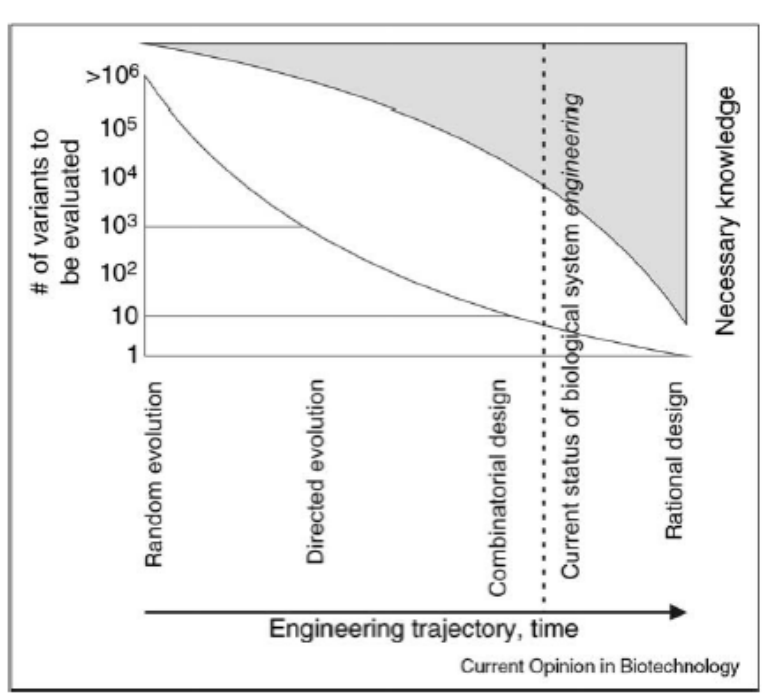

The relationship between required knowledge and different manipulation strategies on the road to engineering design.

Reproduced from Bujara and Panke 2010, 588

By suggesting a continuous progression from "evolutionary" to rational design, this graph stands in a peculiar relation to the main body of Bujara and Panke's paper. The paper speaks primarily to the discontinuous differences between the three modes of iterative design and it does not address at all the growth of knowledge beyond the "current status of biological system engineering." In particular, it does not suggest that the further development e.g. of combinatorial design strategies will produce the knowledge base that would be required for rational design. Indeed, the paper does not even suggest that rational design is more effective than evolutionary design - it appears to be more superior only in being more "rational," that is, in being knowledge-based. ${ }^{24}$ In addition to supplying all these added considerations, the most interesting feature of this graph is that the demands of knowledge loom like a dark cloud above the scene, rendering the image highly ambivalent: On the one hand, it tells a story of progress and a sequence of steps towards the ultimate goal of rational design based on a comprehensive blueprint for optimization. On the other hand, it identifies a daunting demand for knowledge, prompting the reader either to abandon this trajectory with a

\footnotetext{
${ }^{24}$ The graph suggests continuity and thus makes the implicit, albeit highly problematic, assumption that the knowledge required for better ways of running the design cycle is the kind of knowledge that could provide the basis for rational design. Indeed, in their paper Bujara and Panke question that "reducing the complexity of biological systems will facilitate its engineering," commenting that this is only "a hypothesis that still needs to be confirmed in the laboratory" $(2010,589)$. This cautionary remark applies to the reduction of complexity by an increase of knowledge of causal relations and also to its reduction by insulating engineered biological systems from natural ones. If it were possible to run such an experiment, the laboratory test proposed by Bujara and Panke would measure the scientific assignment of priority always to the improvement of causal knowledge against technoscientific requirements of what it takes to achieve effective control. And if the hypothesis would fail to be confirmed, discontinuity would be reestablished.
} 
gloomy outlook on the future of synthetic biology or else to seek out an alternative trajectory that bypasses the need for all this knowledge but accommodates ignorance.

This moment of ambivalence leads to another scene of conflict. Here, anxiety about departing from the respectable path of science becomes transformed into a hopeful image of continuity and a belief in the fusion of opposites. At the beginning of a review of two methods for the more reliable construction of gene circuits one finds a double-pronged credo: "Engineered organisms enable studies of the general organizing principles of life and have the potential to transform industries including medicine, agriculture and energy" (Tabor 2012, 1061). Noting that "[s]ynthetic biologists must often iterate through cycles of optimization when composing even well-understood parts," Jeffrey Tabor welcomes a method that accelerates this design process by offering a standardized "plug-and-play" modification scheme. ${ }^{25}$ Aside from speeding up the achievement of the desired system performance, this method is "also more scalable and amenable to future automation." In the conclusion of his review, Tabor returns to the initial credo not only with respect to the transformation of industries but also in regard to the study of the general organizing principles of life. He could easily make the point now that the design process is a kind of technical probing and as such in and of itself a way of studying these organizing principles of life. However, by "studying" Tabor means something akin to theoretical understanding. Accordingly he first notes a tension between the advance of design methocalcds and the search for true understanding, and then proceeds to dissolve this tension:

"As automated circuit design and assembly dovetails with iterative optimization, our ability to engineer circuits should extend beyond our ability to truly understand how they work. The tractability of modularly constructed synthetic circuits, however, should also feed back to accelerate the cycle of hypothesis generation and testing in systems biology.” (Tabor 2012, 1063)

Along the lines of "what I can create, I can also understand" Tabor proposes that by synthesis engineers learn what it takes to get something to work. Systems biology can then take this up to generate and test hypotheses about the way in which nature gets analogous things to work. He thus arrives at a fusion of design practice and hypothesis testing, but this should not be mistaken for a fusion of technoscientific synthetic biology and scientific notions of truly understanding how things

\footnotetext{
25 Tabor offers an epistemologically telling description of the design-cycle approach: „Here, the first design is based on the ligand-inhibited repressors LacI and TetR. Each is initially placed upstream of an associated fluorescent reporter on a polycistronic mRNA. The operons show poor reporter expression, which is then improved by 'plugging in' additional copies of the appropriate promoter upstream of each reporter. This increases reporter expression, but reveals that the circuit cannot reach the TetR-dominated state. The tetR promoter is then swapped for a stronger version, but this overcompensates for the problem making only the TetR state stable. A library of random tetR ribosome binding sites (RBSs) is then screened, and a variant that hits the bistable sweet spot is found" (Tabor 2012, 1063, compare Litcofsky et al. 2012).
} 
work. After all, when Tabor speaks of the accelerated generation and testing of hypotheses that extend beyond our ability to understand them, he can only be referring to the iterative design process in systems biology that leads to the construction of computer models that step in where humans reach the limits of their ability to understand. Accordingly, Tabor's image of the fusion of gene-circuit construction with the study of organizing principles of life amounts to the construction in parallel of two technical systems, one in vitro or in vivo, the other in silico, each built through a strategic process of generating complexity, such that one can be said to model or instantiate the causal dynamics of the other: It is not the human mind but the computer simulation that "understands" organizing principles of life by learning to model the engineered structures of synthetic biology. Thus, the simulation "understands" these in virtue of resulting from a similar iterative design processes. Accordingly, Tabor's construction of continuity between circuit engineering and achievements of understanding leaves untouched the break with the epistemic values and traditional ideals of science.

\section{(3) The Matter of Definition}

According to Jeffrey Tabor, synthetic and systems biology can exploit how engineered biological devices and engineered computer models inform each other in various ways. This fundamental notion had already found expression in Tabor's student days when he helped produce one of the founding moments of synthetic biology in the popular imagination. He co-designed a roughly $10 \mathrm{~cm}^{2}$ "lawn" of e-coli bacteria that served as a light-sensitive biofilm which produced the message "hello world." Not only is this friendly greeting the first thing that Tabor's bacteria say to us when we ask them to speak, "hello world" are also the first words that computers programmers learn to program and that verify the working of a programming language or computer system. ${ }^{26}$

Quite in the spirit of "hello world," when synthetic biologists aim to construct synthetic organisms, what they do is take biological knowledge, techniques, and parts in order to build up a complex artificial systems that can stand in for natural biological systems. As such, what they are doing with biological tools is what bioinformatics modelers do with computing tools and algorithms, namely build up a complex artificial system that for explorative purposes takes the place of natural biological systems. ${ }^{27}$ Synthetic biology is thus "synthetic" firstly in the sense of not

\footnotetext{
${ }^{26}$ Compare the Wikipedia entry "Hello world program."

27 “[A]s opposed to simulation models transformed into a computational algorithm and run on a digital computer, here the theoretical model rendered as a synthetic model is of the same 'natural kind' as the native networks as well as being embedded in a simulation environment of the 'same materiality,' i.e., the host organism" (Knuutila/Loettgers, 168). Knuutila and Loettgers argue that this supports a "basic-science approach to synthetic biology." However, whether it actually does this or not depends on the question whether one can pick out "theoretical models" as traditionally conceived.
} 
being analytic, of generating rather than reducing complexity, and secondly in the sense of being a non-natural, artificial biology, that is, in virtue of engineering not within the domain of the natural, but entirely within the sphere of the synthetic even as it utilizes knowledge about and materials from the material sphere of the biological. This is what sets it apart from molecular biology as well as genetic engineering. ${ }^{28}$

Classical science or the pursuit to reduce complexity for the purpose of explanation, calculation and mechanistic control assumes the standpoint of an antagonism between mind and world, theory and reality. How can the mind with its limited means and its peculiar demand for human intelligibility forge agreement between its formulae and the infinite variety of appearances? The technosciences in general, and synthetic biology in particular begin in the middle of things, they are right there and on friendly terms ("Hello World") with the world that they squarely inhabit as an extended laboratory which is overflowing with phenomena of their own making (compare Bensaude-Vincent and Simon 2008). These technosciences build on the achievements of science and technology to further enlarge technological and predictive control. Instead of presenting the external world to the human mind, they amalgamate the workings of the human mind with the workings of machinery and the workings of black-boxed biological nature in order to create highly complex, yet reasonably robust structures or processes.

This, then, suggests a definition of synthetic biology that highlights its specific epistemic values and ideals: For constructive purposes synthetic biology builds on the achievement in silico, in vitro, and in vivo of technical control of biological complexity, that is, it is the endeavor of drawing together de facto achievements of technical control for the generation of technical systems with greater biological complexity. ${ }^{29}$

\section{CONCLUSION}

The philosophy of synthetic biology seeks to characterize an emerging, indeed contested field of inquiry. In this survey, it therefore began by showing that different epistemic communities might attach different meanings or interpretations to central concepts such as "understanding," "rational engineering," "evolution," and "design." It was then shown that these different interpretations give

\footnotetext{
${ }^{28}$ Also, this perspective affords a way of distinguishing the simulation approach in synthetic biology from that in systems biology, and thus a way of re-interpreting the examples discussed in Gramelsberger 2013, compare notes 18 and 19 above.

${ }^{29}$ To be sure, ,de facto achievements of technical control of biological complexity“ does not require an understanding of biological complexity, it refers only to the local and partial success stories where some biological process can be manipulated or replicated (in a biological system or in a simulation model).
} 
rise to different stories of how systems biology led to synthetic biology where each of these stories expresses different epistemic values and ideals. But from the tension between scientific and technoscientific epistemic commitments it was still possible to finally distill a definition of synthetic biology. That this was possible is due to the fact that the tension between an engineering approach and the quest for understanding biological processes cannot be resolved in any old way. And pointing this out is a valuable philosophical contribution to synthetic biology at this early stage in its development.

It is quite impossible to simply marry the epistemic ideals of technoscientific synthetic biology to those of biology as a theoretical science - they pull in opposite directions, after all: Here the reduction of complexity for the purposes of intellectual tractability, there the drawing together of scientific knowledge and technological capability for the generation of complexity beyond our ability to truly understand how our own creations work. Here the identification of bottle-necks and needs-to-know for rational engineering, along with the demand for more and better theoretical knowledge in order to diminish ignorance, and there the attempt primarily to discover how much one can achieve even with how little we know, with considerable tolerance for ignorance of everything that can be black-boxed. The tension, even antagonism, between these epistemic ideals cannot be dissolved - which does not preclude, of course, that the corresponding research findings can inform, even inspire one another.

It is not at all impossible, in contrast, to marry the notion of bringing an engineering approach to biology and the notion of knowledge production through synthetic biology - but in order to do so one might have to become dishabituated from the established scientific image of knowledge and of knowledge production. From the point of view of the engineering approach, knowledge and understanding need not be tied to the intellectual tractability of causal relations, nor does it consist in the truth or falsity, or empirical adequacy of linguistic statements such as theories or hypotheses. Instead, knowledge and understanding might reside in computer models and other technologically robust constructions, tied to the iterations of the design cycle as a learning process of sorts.

This allows us finally to appreciate the last sentences of the Nature editorial that appeared on the occasion of Roberta Kwok's analysis, and the tenth anniversary of synthetic biology:

As it develops along this and other paths, synthetic biology itself will demand more by way of new fundamental biological knowledge - quantitative, systematic, computational and biophysical. And conversely, one of the deepest lessons from these first ten years is that biological knowledge will require synthetic approaches if it is to become a mature and reasonably predictive science. (Nature editorial 2010) 
There is little to disagree with in these concluding remarks. To the extent that they gloss over the antagonism between epistemic ideals, these two sentences require only a bit of rephrasing and clarification: Of course, synthetic biology can only benefit from new fundamental biological knowledge - this is an argument for a pluralism of approaches, scientific and technoscientific, within biology. By the same token, synthetic biology will continue in its search for design solutions that do not depend on the availability of new fundamental biological knowledge. And as for the "deepest lesson" offered by synthetic biology, it leads to the question of how the very notion of "biological knowledge" will be transformed through the synthetic approach. This includes the question, for example, of the difference between predicting on the basis of explanatory theories, and predicting on the grounds of technological robustness. With this deepest lesson there is much to do for the philosophy of synthetic biology. ${ }^{30}$

\section{CONTACT}

\section{Alfred Nordmann}

Professor of Philosophy

Technische Universität Darmstadt

Residenzschloss, 64283 Darmstadt, Germany

nordmann@phil.tu-darmstadt.de

\section{REFERENCES}

Adam, Matthias (2010) "Multi-Level Complexities in Technological Development: Competing Strategies for Drug Discovery," in Martin Carrier and Alfred Nordmann (eds.) Science in the Context of Application, Dordrecht: Springer, 2010, pp. 67-83.

Aquinas, Thomas (1986) Von der Wahrheit (De veritate, quaestio I), Hamburg: Meiner.

Benner, Steven, Fei Chen and Zunyi Yang (2011) "Synthetic Biology, Tinkering Biology, and Artificial Biology: A Perspective from Chemistry," in Pier Luigi Luisi and Cristiano Chiarabelli (eds.) Chemical Synthetic Biology, Hoboken: Wiley, pp. 69-106.

Benner, Steven and Michael Sismour (2005) "Synthetic Biology," Nature Reviews Genetics 6: 533-543.

Bensaude-Vincent, Bernadette (2009a) Les Vertiges de la technoscience: Façonner le monde atome par atome, Paris: La Découverte.

Bensaude-Vincent, Bernadette (2009b) "Synthetic biology as a replica of synthetic chemistry? Uses and misuses of history," Biological Theory 4:4, 314-318.

Bensaude-Vincent, Bernadette (2009c) "Biomimetic Chemistry and Synthetic Biology: A two-way traffic across the borders," Hyle 15, 31-46.

Bensaude-Vincent, Bernadette (2013a) "Between the possible and the actual: Philosophical perspectives on the design of synthetic organisms," Futures 3:2, 23-32.

\footnotetext{
${ }^{30}$ For critical comments and suggestions I would like to thank Marta Bertolaso, Jane Calvert, Annamaria Carusi, Bernd
} Giese, Kay Hamacher, Thorsten Kohl, Maureen O’Malley, Eleanore Pauwels, Jan C. Schmidt. 
Bensaude-Vincent, Bernadette (2013b) "Discipline building in Synthetic Biology," Studies in History and Philosophy of Biological and Biomedical Sciences 44:2, 122-129.

Bensaude-Vincent, Bernadette and Jonathan Simon (2008) Chemistry: The Impure Science, London, Imperial College Press.

Breithaupt, Holger (2006) “The engineer's approach to biology,” EMBO Reports 7:1, 21-24.

Bujara, Matthias and Sven Panke (2010) Engineering in complex systems, Current Opinion in Biotechnology 21:586591.

Calvert, Jane (2010) “Synthetic biology: constructing nature?” Sociological Review 58:s1, 95-112.

Canton, Barry, Anna Labno, and Drew Endy (2008) "Refinement and standardization of synthetic biological parts and devices," Nature biotechnology 26:7, 787-793.

Check, Erica (2005) “Designs on Life,” Nature 438:24, 417-118.

Cheng, Allen and Timothy Lu (2012) "Synthetic Biology: An Emerging Engineering Discipline,” Annual Review of Biomedical Engineering 14: 155-178.

Delgado, Ana (2013) “DIYbio: Making things and making futures," Futures: The Journal of Policy, Planning and Future Studies 48, 65-73.

Delgado, Ana and Manuel Porcar (2013) "Designing de novo: interdisciplinary debates in synthetic biology," Systems and Synthetic Biology, 7:1-2, 41-50.

Dyson, Freeman (2007) “Our Biotech Future,” The New York Review of Books 54(12).

Ferber, Dan (2004) “Microbes made to order," Science 303: 158-161.

Forman, Paul (2007) "The Primacy of Science in Modernity, of Technology in Postmodernity, and of Ideology in the History of Technology," History and Technology 23:1/2, 1-152.

Gelfert, Axel (2013) "Synthetic biology between technoscience and thing knowledge," Studies in History and Philosophy of Biological and Biomedical Sciences 44:2, 141-149.

Giese, Bernd, Stefan Koenigstein, Henning Wigger, Jan C. Schmidt, Arnim von Gleich (2013) „Rational Engineering Principles in Synthetic Biology: A Framework for Quantitative Analysis and an Initial Assessment," Biological Theory 8:4, 324-333.

Gleich, Arnim von, Bernd Giese, Stefan Königstein, Jan C. Schmidt (2012) Synthetische Biologie: Revolution oder Evolution? Definition, Charakterisierung und Entwicklungsperspektiven der Synthetischen Biologie mit Fokus auf den damit verbundenen Chancen und Risiken (TAB-Gutachten), Bremen: afortec.

Gramelsberger, Gabriele (2013) "The simulation approach in synthetic biology," Studies in History and Philosophy of Biological and Biomedical Sciences 44:2, 150-157.

Heinemann, Matthias and Sven Panke (2006) "Synthetic biology: Putting engineering into biology," Bioinformatics 22, 2790-2799.

Ideker, Trey, Timothy Galitski and Leroy Hood (2001) “A New Approach to Decoding Life: Systems Biology,” Annual Review of Genomics and Human Genetics 2, 341-372.

Kastenhofer, Karen (2013a) "Synthetic biology as understanding, control, construction, and creation? Techno-epistemic and socio-political implications of different stances in talking and doing technoscience," Futures: The Journal of Policy, Planning and Future Studies 48, 13-22.

Kastenhofer, Karen (2013b) "Two sides of the same coin? The (techno)epistemic cultures of systems and synthetic biology," Studies in History and Philosophy of Biological and Biomedical Sciences 44, 130-140.

Kitano, Hiroaki (2002) “Systems Biology: A Brief Overview,” Science 295: 1662-1664.

Kitano, Hiroaki (2004) “Biological Robustness,” Nature Reviews Genetics 5, 826-837. 
Knuuttila, Tarja and Andrea Loettgers (2013) "Basic science through engineering: Synthetic modeling and the idea of biology-inspired engineering," Studies in History and Philosophy of Biological and Biomedical Sciences, 44:2, 158-169.

Kwok, Roberta (2010) "Five Hard Truths for Synthetic Biology,” Nature 463, 288-290.

Lenhard, Johannes and Eric Winsberg (2010) "Holism, entrenchment, and the future of climate model pluralism," Studies in History and Philosophy of Modern Physics 41, 253-262.

Levskaya, Anselm, Aaron Chevalier, Jeffrey Tabor, Zachary Simpson, Laura Lavery, Matthew Levy, Eric Davidson, Alexander Scouras, Andrew Ellington, Edward Marcotte, Christopher Voigt (2005) "Synthetic biology: Engineering Escherichia coli to see light," Nature 438, 441-442.

Lewens, Tim (2013) "From bricolage to BioBricks ${ }^{\mathrm{TM}}$ : Synthetic biology and rational design," Studies in History and Philosophy of Biological and Biomedical Sciences 44, 641-648.

Litcofsky, Kevin, Raffi Afeyan, Russell Krom, Ahmad Khalil, James Collins (2012) "Iterative plug-and-play methodology for constructing and modifying synthetic gene networks," Nature Methods 9, 1077-1080.

MacLeod, Miles and Nancy Nersessian (2013) "Building Simulations from the Ground Up: Modeling and Theory in Systems Biology," Philosophy of Science 80, 533-556.

Mast, Christof, Friederike Möller and Dieter Braun (2013) „Lebendiges Nichtgleichgewicht: Unter welchen physikalischen Randbedingungen kann Leben entstehe?“ Physik Journal 12:10, 29-35.

Nature editorial (2010) “Ten years of synergy,” Nature 463, 269-270.

Nordmann, Alfred (2010a) „Enhancing Material Nature. (In Kamilla Lein Kjølberg and Fern Wickson (eds.) Nano meets Macro: Social Perspectives on Nanoscale Sciences and Technologies, Singapore: Pan Stanford, 2010 , pp. 283-306.

Nordmann, Alfred (2010b) "Science in the Context of Technology," in Martin Carrier and Alfred Nordmann (eds.) Science in the Context of Application, Dordrecht: Springer, 2010, pp. 467-482.

O’Malley, Maureen (2009) “Making Knowledge in Synthetic Biology: Design Meets Kludge,” Biological Theory 4:4, 378-389.

O’Malley, Maureen (2011) "Exploration, iterativity and kludging in synthetic biology," Comptes Rendus Chimie 14:4, 406-412.

O’Malley, Maureen and John Dupre (2005) “Fundamental issues in systems biology,” BioEssays 27, 1270-1276.

O’Malley, Maureen, Alexander Powell, Jonathan F. Davies, Jane Calvert (2008) "Knowledge-making distinctions in synthetic biology," BioEssays 30, 57-65.

Pauly, Philip (1987) Controlling Life: Jacques Loeb and the Engineering Ideal in Biology, New York: Oxford University Press.

Rodrigo, Guillermo, Thomas Landrain, and Alfonso Jaramillo (2012) "De novo automated design of small RNA circuits for engineering synthetic riboregulation in living cells," Proceedings of the National Academy of Sciences 109:38, 15271-15276.

Rollié, Sascha, Michael Mangold, and Kai Sundmacher (2012) "Designing Biological Systems: Systems Engineering meets Synthetic Biology," Chemical Engineering Science 69:1, 1-29.

Royal Academy of Engineering (2009) Synthetic Biology: scope, applications and implications, London: Royal Academy of Engineering.

Schmidt, Jan. C. (this volume) "Synthetic Biology as Late-Modern Technology"

Schmidt, Markus (2009) "Do I understand what I can create? Biosafety Issues in Synthetic Biology," Markus Schmidt, Alexander Kelle, Agomoni Ganguli-Mitra, Huib de Vriend (eds.) Synthetic Biology: the technoscience and its societal consequences, Dordrecht: Springer, 81-100.

Smith, Pamela (2004) The Body of the Artisan: Art and Experience in the Scientific Revolution, Chicago: University of Chicago Press. 
Schyfter, Pablo (2013) "How a 'drive to make' shapes synthetic biology," Studies in History and Philosophy of Biological and Biomedical Sciences 44, 632-640.

Schummer, Joachim (2011) Das Gotteshandwerk: Die künstliche Herstellung von Leben im Labor. Berlin: Suhrkamp.

Tabor, Jeffrey (2012) Modular gene-circuit design takes two steps forward, Nature Methods 9:11, 1061-1063.

Tal, Eran (2013) "Enhancing Knowledge, Affording Ignorance," Presentation at Conference What Affordance Affords, Darmstadt, November 26.

UK Synthetic Biology Roadmap Coordination Group (2012) A Synthetic Biology Roadmap for the UK, Swindon: Technology Strategy Board.

Vico, Giambattista (1979) Liber Metaphysicus, Munich: Wilhelm Fink Verlag.

Wolkenhauer, Olaf and Mihajlo Mesarovic (2005) „Feedback dynamics and cell function: Why systems biology is called Systems Biology," Molecular BioSystems 1, 14-16. 